\title{
Research on the application situation and its architecture of the Internet of things
}

\author{
Dongjuan Gu, Lei Xia
}

Nanchang institute of science \& technology, China

Keywords: Internet of things, application technology, architecture

\begin{abstract}
The Internet of things makes the physical world of human existence networking and informatization, integrates the separated physical world and information space interconnection, is the development trend of future network. On the basis of introducing the basic concept and development situation of the Internet of things, this paper gave some successful application cases of the Internet of things in some basic fields, and analyzed the architecture and related technologies of the Internet of things.
\end{abstract}

\section{Introduction}

The Internet of things is known as the new wave of the world information industry after the computer, Internet and mobile communication network. The Auto-ID center at the Massachusetts institute of technology for the first time defined the Internet of things as: "the network connects all items with the Internet through radio frequency identification and bar code information sensing equipment to realize the intelligent identification and management functions" [1].

International telecommunication union formally put forward the definition of the Internet of things in the "ITU Internet report 2005: the Internet of things": "Through the information sensing devices, such as radio frequency identification, sensors, GPS, laser scanners and so on, to make the "objects" with automatic marking and intelligent perception ability, to achieve the connection of the physical world and the virtual digital world. Through the intelligent interface of the "objects" to achieve seamless integration with information network, through the exchange of information and communication, so as to achieve the intelligent identification, positioning, tracking, monitoring and management, and ultimately achieve the interconnection between any time, any place and any object, to realize the ubiquitous network and the ubiquitous computing "[2].

\section{The application practice of the Internet of things}

In today's world, all countries have carried out a strategic plan for the development of the Internet of things, such as America's "smart grid", "smart earth", "smart dust", etc. And our country has listed the sensor network into key research areas in the major projects of "the national medium and long-term science and technology development plan (2006-2020)" and "a new generation of broadband mobile radio communication network", and set up the projects of National Natural Science Fund, such as "key technology research of the security for the Internet of things", the network technology development strategy research for the future of Internet", "collaborative control research based on object perception driven under the environment of the Internet of things" and so on[3].

With the approval of the State Council "the report on the support of the construction for the national innovation demonstration zone (National sensor information center) in Wuxi" in November 2009, the telecom operators have also given a high degree of attention for the Internet of things industry in our country. China mobile communications group and Wuxi city government achieved the strategic cooperation intention for promoting the integration of TD-SCDMA and the Internet of things, and set up a China Mobile Wuxi Institute of Internet of things. China telecom group cooperated with the people's government in Wuxi set up the application and promotion center of China telecom Internet of things and the key technology research laboratory of Internet of things in Wuxi city. China unicom founded the institute of Internet of things and the technology application 
development and research center of the institute for Internet of things, cloud computing center, disaster recovery and emergency center, and set up "National Engineering Laboratory of the application for the next generation Internet broadband service in Wuxi. National grid set up technology center of smart grid information and communication in Wuxi and so on. With the deepening of the research and the gradually maturation of technical scheme, the Internet of things has been advancing in the fields of medical and health undertakings, the management of agriculture and animal husbandry, construction engineering, environmental monitoring, electronic commerce, national public security, etc.

\subsection{Medical and health undertakings}

In the field of medical and health services, the Internet of things technology is mainly used in medicine, health and food safety and other aspects. The Internet of things technology is used in the whole medical process for the patients, such as adopts electronic medical communication, effectively shorten the registration time of patients, especially the critical patients, and the medical staff can check the database, accurate access to the patient's physical condition, past medical history and treatment and other related information. At the same time, with the integration of sensing technology, the medical staff can use wristbands with sensors to achieve real-time monitoring of the patients. With the support of large data, the Internet of things technology also can be used in the production of food and drugs, anti-counterfeiting, circulation management and other aspects, to achieve the traceability of quality.

\subsection{The management of agriculture and animal husbandry}

The application of the Internet of things in agricultural mainly includes the application on the aspects of agricultural production, animal source, greenhouse control, forest fire prevention, etc. In the aspect of agricultural production, adopts the intelligent sensor technology to obtain the environmental information, such as solar effective radiation, soil moisture, compactness, agricultural machinery, oil consumption, speed, etc., through the transmission way of operation support platform and GPRS/TD network, using various means to transmit the heterogeneous sensor data to the server and security center, and to analyze it and find the solutions, and then will be applied to food production, agricultural machinery scheduling, resource monitoring, etc.

\subsection{Construction engineering}

The application of Internet of things technology in construction engineering is mainly manifested in the use of sensing technology to realize the data collection of terminal, after transferred to the service platform, can carry out the management of monitoring and control for relevant engineering and projects. Such as the road lighting system based on Internet of things realizes its own network by embedding the wireless communication module in each terminal, accepts the command from the control center and feeds back the status of the street lamp to the control center. Control box uses ZigBee technology and all terminal communications under the jurisdiction of the road, uses GPRS to communicate with control center, and according to the instructions or time of control center and the brightness of sunshine to issue the control command for each lamp, automatically adjusts the power balance of the whole road. Mobile computing tools (notebook computer, PDA, mobile phone) and street lights maintenance vehicle can also through the control center for remote telemetry and remote control [4]. China mobile also implements the terminal automatic alarm, anti-theft alarm, all kinds of data collection and analysis, etc on the basis of "wireless street lamp control solutions".

In addition: China telecom "family Internet of things system" includes smart household appliances, articles for daily use, household equipment, home health equipment, and so on, these equipment can be equipped with sensors, RFID and RF read-write device, which can realize the condition monitoring of household goods and household equipment, the health data collection of family members, etc. Collected data through the broadband network and family Internet of things platform to be transferred to the application platform of the Internet of things, such as market, manufacturers, medical institutions, etc, to realize the detection, control and distribution of household items, provide the after-sales service tracking, and provide the health services for family members timely, etc. 


\section{The system of the Internet of things}

Combined with the above application cases, the Internet of things usually includes information acquisition, transmission and processing, the whole system can be divided into four parts: the perception layer, the transport layer, the support layer and the application layer. The architecture of

the Internet of things as shown in figure 1:

\subsection{Perception layer}

Figure 1 The architecture of Internet of things technology

The perception layer is used to perceive and identify the information of the specific objects (such as material properties, environment status, behavior trend, and other dynamic and static information), includes sensors and sensor networks/gateway, two-dimensional code label and tag reader, RFID tags and tag reader, camera, GPS, SIM card, and so on, the involved key technologies include sensor technology, micro-electromechanical system, multi-label multi-reader anti-collision technology, short distance networking technology.

Because of the perception layer located in the source position of the whole system, therefore, it is required to have an accurate perception, and to meet the low power consumption, miniaturization, high reliability, low cost, at the same time, in the long run, we should focus on national standardization construction and the research and development and breakthrough of key technology.

\subsection{Transport layer}

The function of the transport layer is data interaction and transmission, the carrier can be broadband Internet, cable access network or wireless mobile communication network, such as 2G/3G/4G communication technology, NGN technology, heterogeneous network integration technology, information encoding/ authentication technology, etc. Relatively, wireless mobile communication network has irreplaceable advantages with coverage and convenience, etc. At the same time, in the architecture of the Internet of things, the standardization degree is highest in the transport layer, and the application is also the most mature.

\subsection{Support layer}

Under the support of high performance computing technology, support layer integrates the large and huge amounts of information resources in the network through calculation and analysis into a large intelligent network which can be interconnected, to set up an efficient, reliable and credible support technology platform for the upper layer service management and the large-scale industry application. Such as through the center computer with super powerful capacity, storage cluster and intelligent information processing technology, the massive information in the network can be processed in real time with high speed, and the data can be intelligently mined, managed, controlled and stored at any time.

\subsection{Application layer}

Application layer according to the needs of users, build the management platform and running platform of the oriented application faced to all kinds of industry, and according to the 
characteristics of the various application to integrate related services. In order to provide accurate information services, must be combined with professional knowledge and business models of different industries, to complete a more detailed and accurate intelligent information management. Especially in the aspects of networking software and application software, existing software can't simply be applied directly to the environment of the Internet of things, needs to research, design and develop the network access and network application platform software with high stability and high reliability on the basis of the introduction of the concept for control loop [5].

The various levels of the Internet of things not only both independent, but also close link, in order to achieve the optimization function of the overall system, service for a particular application, the resource need to be co-allocated and shared between each layer. The synergistic treatment of convergence perception and the Internet of things based on sensor network, the full integration of mobile communication system and wireless sensor network will form a more extensive network, it will achieve any time, any place, any person, any thing all can be smoothly communicated, and with the basic characteristic of omnipresence, everything is contained and omnipotence to form the ubiquitous network, to provide more high-quality industry project scheme and more convenient service experience for the users.

\section{Conclusion}

The application program of the Internet of things technology includes five key elements, namely: can access network intelligent equipment, digital management equipment, application support and operating platform[6]. Mobile communications network with the characteristics of wide coverage, mature application technology, high industry standardization degree, rich terminal in each layer, etc, to become the medium which is more easy to implement the transmission, control and management of Internet of things. Mobile network base station, mobile phones and other intelligent terminals all can be referred to as the sensor node which is used to perceive the information acquisition. Mobile communication operators have rich experiences on network equipment, business achievement, business management, user management, information security and so on, the presence of these existing resources makes the architecture of the Internet of things more secure and healthy.

\section{References}

[1] Yunhao Liu. The introduction of the Internet of things [M]. Beijing: science press, 2010:1-6.

[2] ITU Strategy and Policy Unit(SPU). ITU internet reports 2005:the Internet of things[R]. Geneva: International Tele-communication Union(ITU), 2005.

[3] The project application and funding of national natural science fund [EB/OL]. [2011-01-06]. HTTP://www.nsfc.gov.cn/nsfc/cen/xxgk/slzz.html.

[4] Min Pu, Yunfei Li, Yihuai Wang. Wireless lighting control system based on Internet of things [J]. Journal of lighting engineering, 2010 (2) : 86-89.

[5] Feizhou Zhang, Dongkai Yang, Zhi Chen. The introduction of the Internet of things technology[M]. Beijing: electronic industry press, 2010:187-190.

[6] The Internet of things in China. Research report on the development of the Internet of things in 2010. [EB/OL]. [2010-07-02]. HTTP://m2m.wuxi.gov.cn/cyyy/yjcg/561313_3.shtml. 